Imperial College London, London, United Kingdom: ${ }^{3}$ Cardiovascular Division, King's College London, British Heart Foundation Centre, London, United Kingdom; ${ }^{4}$ HistologyEmbryology Department, Faculty of Medicine, University of Athens, Athens, Greece

10.1136/thoraxjnl-2013-204457.5

Introduction Cardiovascular disease (CVD) is a major cause of death in smokers, particularly in patients with chronic obstructive pulmonary disease (COPD). Circulating endothelial progenitor cells (EPC) are required for endothelial homeostasis, and their dysfunction contributes to CVD. DNA damage has been recognised as an important contributor to CVD. Our aim was to investigate whether EPC from smokers and COPD patients are dysfunctional, and the role of DNA damage pathways in mediating endothelial dysfunction in these patients.

Methods To investigate EPC dysfunction in smokers, we isolated and expanded blood outgrowth endothelial cells (BOEC) from peripheral blood samples of healthy non-smokers, healthy smokers and COPD patients. Endothelial senescence was measured by senescence-associated $\beta$-galactosidase (SA- $\beta$-Gal) activity. Expression of sirtuin (SIRT)-1, p16, p21, $\gamma$-H2AX and 53BP1 were measured by Western blotting and/or immunofluorescence confocal microscopy. SIRT1 activity was measured using a SIRT1 fluorescent activity assay kit. To investigate angiogenesis in vivo, BOEC were labelled with Vybrant DiI Cell-Labelling Solution, mixed with Matrigel and injected subcutaneously into the back of NOD. CB17-Prkdcscid/NcrCrl mice. Seven days later, the mice were sacrificed and the plugs were cryosectioned.

Results BOEC from smokers and COPD patients showed increased DNA double-strand breaks (measured by $\gamma-\mathrm{H} 2 \mathrm{AX}$, 53BP1) and senescence (senescence associated- $\beta$-galactosidase activity, p16 and p21 levels) compared to non-smokers. Senescence negatively correlated with sirtuin-1 (SIRT1) expression and activity, a protein deacetylase that inhibits DNA damage and cellular senescence. Inhibition of DNA damage response by silencing of ataxia telangiectasia-mutated (ATM) kinase resulted in up-regulation of SIRT1 expression and decreased senescence. Interestingly, treatment of BOEC from COPD patients with the SIRT1 activator resveratrol or a selective ATM inhibitor rescued the senescent phenotype. Using the in vivo Matrigel plug assay, BOEC from COPD patients displayed reduced angiogenesis (capillary-like structures) and increased DNA damage, senescence and apoptosis (measured by 53BP1, p16, TUNEL and cleavedcaspase 3 staining) compared to non-smokers.

Conclusions BOEC from smokers and COPD patients show reduced angiogenesis in vivo and display increased DNA damage and senescence, associated with reduced SIRT1 expression. These defects may contribute to endothelial dysfunction and cardiovascular events in smokers and COPD patients and could potentially constitute therapeutic targets for intervention.

\section{T6 THE ROLE OF IL-17A IN A MOUSE MODEL OF PULMONARY INFECTION CAUSED BY STREPTOCOCCUS PNEUMONIAE IS STRAIN DEPENDENT}

${ }^{1}$ ND Ritchie, ${ }^{2}$ TJ Mitchell, ${ }^{1} \mathrm{TJ}$ Evans; ${ }^{1}$ University of Glasgow, Glasgow, United Kingdom; ${ }^{2}$ University of Birmingham, Birmingham, United KIngdom

\subsection{6/thoraxjnl-2013-204457.6}

Background The cytokines interleukin 17A and interleukin 22 are known to be important in host defence against Gram-negative infection. However, their role in the innate immune response to pneumococci remains poorly understood. We aimed to investigate the role of these cytokines in an animal model of pneumococcal infection.

Methods Wild type (WT), IL-17 receptor A-/- (IL17RAKO) and IL-22-/- C57Bl/6 mice were infected intranasally with serotype 4 (TIGR4) and serotype 3 (SRL1) pneumococci. Neutrophils for in vitro experiments were obtained by peritoneal lavage following injection of casein.

Results TIGR4 was cleared from the lungs of mice within 48 hours but invaded early in the course of the infection causing bacteraemia and disseminated infection. In contrast, SRL1 invaded into blood late in the course of infection but caused a dense consolidative pneumonia with purulent empyema. IL-17A and IL-22 were detected in alveolar lavage within 6 hours of infection but fell in later infection suggesting an innate source. Contrasting results were obtained when knockout animals were infected. In TIGR4 infection, IL17RAKO were more susceptible to infection $(P=0.04)$ and this was due to an increased incidence of early bacteraemia in these animals. In contrast, following SRL1 infection in IL17RAKO animals, there was improved survival $(P=0.004)$. In both types of infection, IL22KO animals demonstrated an intermediate phenotype that did not reach statistical significance. IL17RAKO mice had decreased neutrophils in blood and lung within 24 hours of infection in keeping with the known biological actions of IL-17A. Studies using isolated cells showed that TIGR4 was phagocytosed and killed by neutrophils but uptake and killing of SRL1 was ineffective. Experiments with fluorescent bacteria confirmed poor neutrophil uptake of SRL1 relative to TIGR4. Depletion of WT mouse neutrophils with a monoclonal antibody demonstrated a trend towards delayed mortality and improved outcome in SRL1 infection. 